\title{
Automatic Quantification of DTI Parameters along Fiber Bundles
}

\author{
Jan Klein, Simon Hermann, Olaf Konrad, Horst K. Hahn, \\ Heinz-Otto Peitgen \\ MeVis Research, 28359 Bremen \\ Email: klein@mevis.de
}

\begin{abstract}
We introduce a novel technique that allows for an automatic quantification of MR DTI parameters along arbitrarily oriented fiber bundles. Most previous methods require either a manual placement of ROIs, are limited to single fiber tracts, or are limited to bundles which are perpendicular to one of the three image planes. Thus, the quantification process is made much more time-efficient and robust by our new approach. We compare our technique with a manual quantification of an expert and show the similarity of the results. Furthermore, we demonstrate how to visualize the parameters at a certain position of the fiber bundle so that areas of interest can easily be examined.
\end{abstract}

\section{Introduction}

Over the last few years, diffusion tensor imaging (DTI) received increasing attention in the neurosurgical and neurological community with the motivation to identify white matter tracts afflicted by an individual pathology or tracts at risk for a given surgical approach [1]. An explicit geometrical reconstruction of white matter tracts has become available by fiber tracking based on DTI data [2].

Color-coded maps of fractional anisotropy (FA) computed from DTI data were successfully employed in several studies where it has been shown that modified parameters like FA, relative anisotropy or diffusion strength are an indicator of diseases affecting white matter tissue $[3,4,5]$.

A pointwise assessment of those parameters was proposed along a single streamline combined with a visualization of uncertainties in [6]. Other quantification algorithms $[4,3,5]$ use a manual definition of one or more ROIs which cover a certain fiber bundle at some slices of the image data. Within such ROIs, more robust parameters can be computed from several single parameters. In a previous work, we extended standard ROI-based techniques by considering partial volume effects so that fibrous and non-fibrous tissue can be classified [7]. Recently, it has been shown how to define fiber bundles implicitly and how to compute their parameters as integrals [8].

However, it is often desirable to determine the parameters along a whole fiber bundle as precisely as possible so that several ROIs have to be drawn manually via a multi-planar reconstruction. As this process is very time-consuming and 
error-prone, [9] proposed a semi-automatic algorithm where ROIs are placed on axial, sagittal, or coronal slices between two predefined ROIs automatically. As a consequence, reliable results can only be achieved if the fiber tracts are perpendicular to one of the three image planes. Moreover, curved fiber bundles cannot be handled by their approach and had to be divided into several parts.

\section{$1.1 \quad$ Novel contributions}

In this paper, we propose a method that overcomes the problems mentioned above and that allows for an efficient and automatic quantification of MR DTI data along arbitrarily oriented fiber tracts. A parameter map, defined by a Delaunay triangulation, visualizes the distribution of all single data points contributing to one single parameter. In contrast to the method proposed by Fillard [10], we do not compute an average parameter by several single values that all have the same geodesic distance from a user-defined origin (ROI). Instead, we compute a reference plane depending on the local curvature of the fiber bundle, which is used afterwards to determine the nearest fiber points with corresponding parameters. Thus, more reasonable results may be achieved by our new approach when dealing with geometrically complex fiber bundles.

\section{Methods}

For a tumor patient suffering from a right hemispheric glioma (F, 73 y), echoplanar DTI data were acquired on a $1.5 \mathrm{~T}$ Siemens Sonata (image resolution $1.875 \times 1.875 \times 1.9 \mathrm{~mm}^{3}, 60$ slices, 6 gradient directions). Using a deflectionbased algorithm [11], we compute the fiber bundle which has to be quantified. During fiber tracking, the parameters of interest (e.g., the FA) are computed and stored at each fiber point. We developed a simple filtering tool, where fibers can be excluded from the bundle or can be cropped at their endings afterwards.

\subsection{Quantification}

For quantification, two steps have to be done. First, each fiber is resampled so that all fibers consist of the same number of equidistantly distributed fiber points as proposed in [12]. Using the resampled fibers, an average center line of all fibers is computed where its $j$-th point $p_{j}$ is calculated by averaging all $j$-th points of all fibers: $p_{j}=\frac{\sum_{i=1}^{n} f_{i}(j)}{n}$. This center line yields to $n$ reference planes $h_{j}:\left(\vec{x}-\overrightarrow{p_{j}}\right) \cdot\left(\overrightarrow{p_{j}}-\overrightarrow{p_{j+1}}\right)=0$ which are used for the quantification.

In a second step, for each point of the center line, a corresponding quantification parameter $v_{j}$ can be determined by $v_{j}=g\left(v\left(f_{1}(j)\right), \ldots, v\left(f_{n}(j)\right)\right)$. There, $v\left(f_{i}(j)\right)$ denotes a single parameter corresponding to fiber point $f_{i}(j)$, and $g$ denotes an arbitrary function with $n$ input parameters, e.g., it computes the average of the $n$ values. However, there may be some outliers $f_{i}(j)$ which are far away from the plane $h_{j}$ (Fig. 2). In this case, $v_{j}$ would also be determined depending on unwanted outliers $v\left(f_{i}(j)\right)$. Thus, we propose to replace them by $v\left(f_{i}(k)\right)$ where $f_{i}(k)$ is the point with minimum distance to the plane $h_{j}$. 
Fig. 1. We compared our new automatic quantification technique with our manual quantification [7]. (i): the reference plane (visualized as orange circle) is automatically determined by our new technique, $F A=0.421$. (ii): a ROI has to be drawn manually within our manual quantification tool, $\mathrm{FA}=0.464$

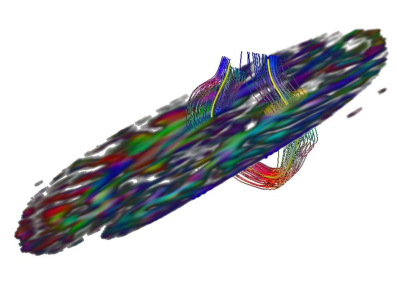

(i)

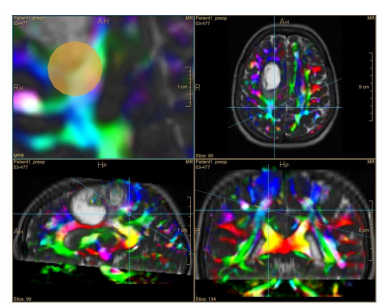

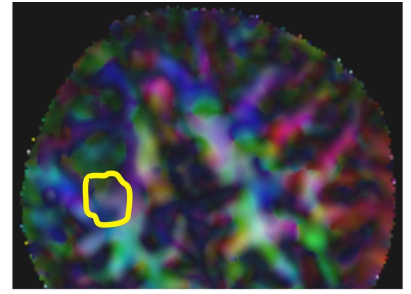

(ii)

\subsection{Visualization}

For visualizing all parameters contributing to $v_{j}$, we perform a Delaunay triangulation of the corresponding fiber points. The resulting triangles are shaded depending on the parameters $v\left(f_{i}(j)\right)$. Simultaneously, the reference plane $h_{j}$ and the fiber bundle are displayed in $3 \mathrm{D}$. The user can interactively slide through the fiber bundle so that the parameter map and the plane are updated (Fig. 3).

\section{Results}

A fiber bundle, which is part of the corpus callosum and consists of $n=50$ single fibers, was tracked and cropped at it endings (Fig. 3). The maximum number of fiber points along a fiber is 1183 so that we determined 1183 average, minimum, and maximum FA values, each of them computed by about 50 single parameter values. The results can be found in Fig. 3 (right). The whole computation including the center line and all FA values took much less than a second (AMD Athlon 64 X2 Dual, 3800+). Fig. 2 shows the distribution of all 50 parameters contributing to $v_{j}$ (the plane $h_{j}$ was chosen as displayed at the bottom of Fig. 3).

We also compared our technique with our manual quantification tool [7] which considers partial volume effects and which performs a classification of fibrous as well as non-fibrous tissue before the quantification. For several regions, we measured the average FA values by both methods, an example is shown in Fig. 1. The difference was always smaller than 0.05 .

\section{Discussion}

Compared to a manual quantification, which takes at least 30 minutes for a reasonably detailed measurement of a fiber bundle (> 50 measured parameters), our new approach drastically reduces the time to a few milliseconds. For a fair comparison we have to mention that for a manual quantification the fiber tracking as well as the cropping and filtering (which takes us about one minute) have not to be done explicitly, but only implicitly by defining the ROIs. 
Fig. 2. Left: there may be some outliers $f_{i}(j)$ which are far away from the plane $h_{j}$ used for computing the quantification parameter $v_{j}$. In this case, we substitute $v\left(f_{i}(j)\right)$ by $v\left(f_{i}(k)\right)$ where $f_{i}(k)$ has the minimum distance to $h_{j}$. Right: Delaunay triangulation indicating the distribution of the single parameters (yellow) used for computing $v_{j}$ (blue: low FA, red: high FA)
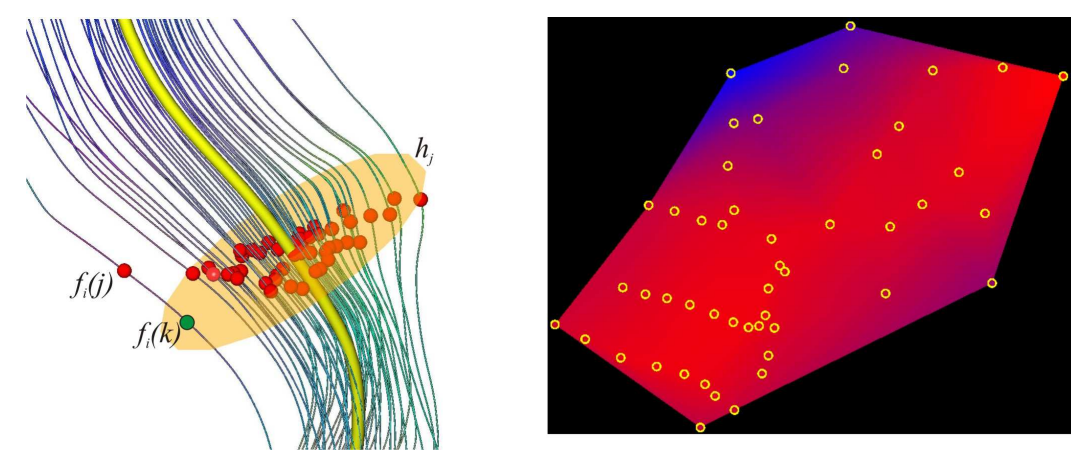

Initial experiments have shown that the differences between the manual and our new automatic quantification are at most 0.05 . They may be explained by the manual determination of the plane, which is used for placing the ROI, as well as by the different algorithmic computation of the parameter values: the manual quantification needs a more complex function for computing the average FA value within an ROI, because it has to differentiate between FA values belonging to fibrous and to non-fibrous tissue. In contrast, this has not to be done by our novel approach because the preceding fiber tracking solves the problem so that only a simple function $g$ depending on $n$ input parameters can be used for the computation (see Section 2). In the future, we would like to use the idea of moving least squares for computing the center line. Furthermore, a more rigorous comparison between manual and automatic quantification has to be done depending on different data sets.

\section{References}

1. Yamada K, Kizu O, Mori S, et al. Brain fiber tracking with clinically feasible diffusion-tensor MR imaging: initial experience. Radiology 2003;227(1):295-301.

2. Mori S, Crain BJ, Chacko VP, et al. Three-dimensional tracking of axonal projections in the brain by magnetic resonance imaging. Ann Neurol 1999;45(2):265-269.

3. Tievsky AL, Ptak T, Farkas J. Investigation of apparent diffusion coefficient and diffusion tensor anisotropy in acute and chronic multiple sclerosis lesions. Am J Neuroradiol 1999;20(8):1491-1499.

4. Stahl R, Dietrich O, Teipel S, et al. Assessment of axonal degeneration on alzheimer's disease with diffusion tensor MRI. Radiologe 2003;43(7):566-575.

5. Tropine A, Vucurevic G, Delani P, et al. Contribution of diffusion tensor imaging to delineation of gliomas and glioblastomas. J Magn Reson Imaging 2004;20(6):905912.

6. Jones DK, Travis AR, Eden G, et al. PASTA: Pointwise assessment of streamline tractography attributes. Magn Reson Med 2005;53:1462-1467. 
Fig. 3. Left: fiber bundle that we use for quantification. The center line is shown in yellow. Right: plot which shows maximum, minimum and average FA values along the fiber bundle. The vertical line corresponds to the orange plane $h_{j}$ shown on the left
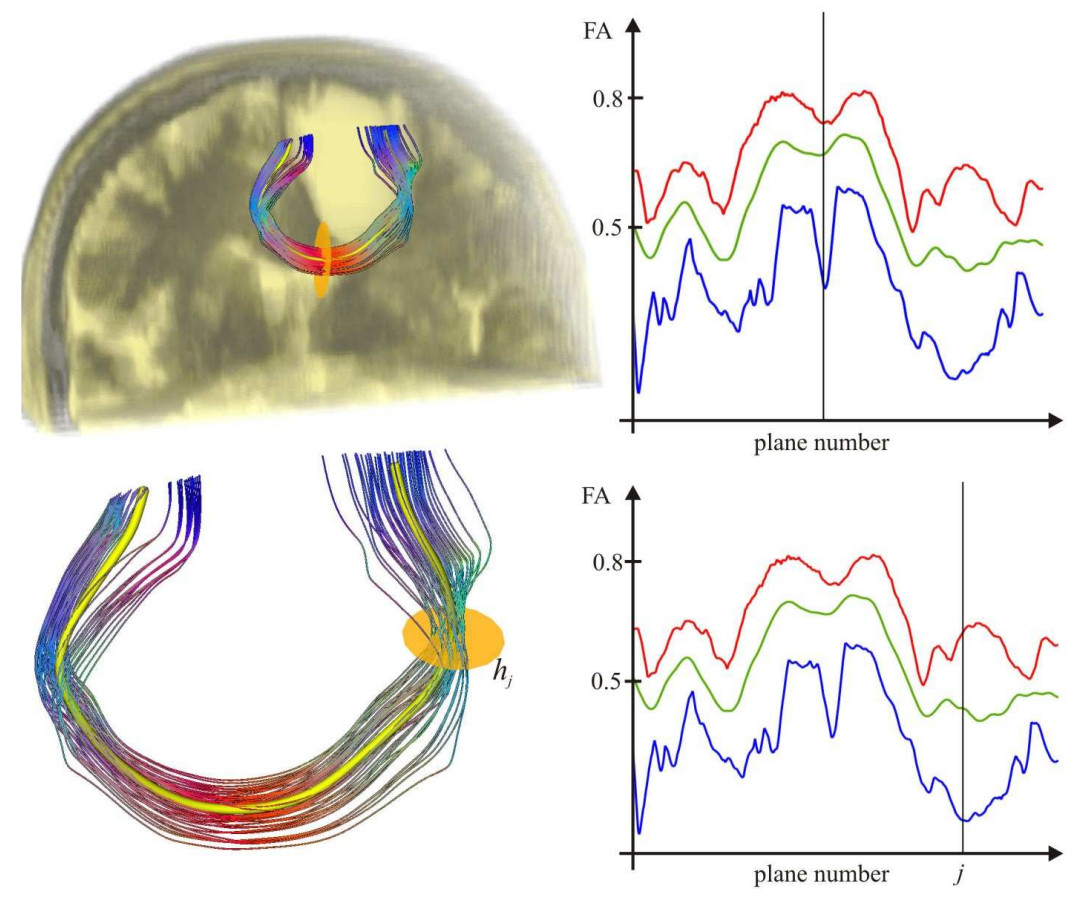

7. Schlüter M, Stieltjes B, Hahn HK, et al. Detection of tumour infiltration in axonal fibre bundles using diffusion tensor imaging. Int J Medical Robotics and Computer Assisted Surgery 2005;1:80-86.

8. Niethammer M, Bouix S, Westin CF, et al. Fiber bundle estimation and parametrization. In: Procs MICCAI'06; 2006. 252-259.

9. Aoki S, Iwata NK, Masutani Y, et al. Quantitative evaluation of the pyramidal tract segmented by diffusion tensor tractography: feasibility study in patients with amyotrophic lateral sclerosis. Radiation Medicine 2005;23(3):195-199.

10. Fillard P, Gilmore J, Lin W, et al. Quantitative analysis of white matter fiber properties along geodesic paths. In: Procs MICCAI'03; 2003. 16-23.

11. Schlüter M, Konrad O, Hahn HK, et al. White matter lesion phantom for diffusion tensor data and its application to the assessment of fiber tracking. Procs SPIE $2005 ; 5746: 835-844$.

12. Enders F, Sauber N, Merhof D, et al. Visualization of white matter tracts with wrapped streamlines. In: Provs IEEE Visualization; 2005. 51-58. 